\title{
准分子激光改变 $\mathbf{C}_{60}$ 薄膜电导率
}

\author{
宁 东 楼祺洪 董景星 魏运荣 向世清 \\ (中国科学院上海光学精密机械研究所, 上海 201800)
}

\section{关键词 准分子激光 $\mathbf{C}_{60}$ 薄膜 电导率 Raman 谱}

利用紫外激光直接改变材料电导率, 具有广泛的应用前景, 现正日益引起人们的重视. 目 前, 在这一领域的研究大多集中于各种有机高分子聚合物 ${ }^{[1]}, \mathrm{C}_{60}$ 是最近才出现的新材料. 根 据能带理论计算, $\mathrm{C}_{60}$ 晶体为典型的直接能隙半导体, 其能隙宽度为 $1.5 \mathrm{eV}^{[2]}$. 但多晶 $\mathrm{C}_{60}$ 薄 膜却是良好的绝缘体, 其本正电导率低于 $10^{-7} \mathrm{~S} \cdot \mathrm{cm}^{-1[3]}$. 最近, Phillips 等报道了用 $248 \mathrm{~nm}$ 的 $\mathrm{KrF}$ 准分子激光提高 $\mathrm{C}_{60}$ 薄膜电导率的实验研究, 并提出电导率变化的主要机制是激光诱 导的绝缘体 - 金属相变 ${ }^{[4]}$.

本文进行了准分子激光诱导 $\mathrm{C}_{60}$ 薄膜电导率提高的实验研究. 在低于刻蚀阈值的激光脉冲照 射下, $\mathrm{C}_{60}$ 薄膜电导率提高了 6 个数量级. Raman 谱的研究表明: 电导率变化的主要机制是 $\mathrm{C}_{60}$ 被 光致氧化分解为无定形碳, 在较低能流密度的情况下, 观察到微晶石墨及 $\mathrm{C}_{60}$ 聚合团簇的形成.

\section{1 实验}

薄膜样品由纯度 $99 \%$ 的 $\mathrm{C}_{60}$ 多晶粉末真空蒸镀于 $15 \mathrm{~mm} \times 15 \mathrm{~mm}$ 的石英基片, 蒸发温度 $400 \sim 450{ }^{\circ} \mathrm{C}$. 由于电导实时测量的需要, 在基片上预铰了两条 $12 \mathrm{~mm} \times 3 \mathrm{~mm}$, 间隔 $1 \mathrm{~mm}$ 的 $\mathrm{Ag} / \mathrm{C}$ 电极, 测量导线用银导电胶粘接到电极一端. $\mathrm{C}_{60}$ 薄膜大小约 $5 \mathrm{~mm} \times 5 \mathrm{~mm}$. 用干涉测量仪 和台阶扫描仪测得电极厚度为 $600 \mathrm{~nm}\left(\mathrm{Ag}\right.$ : $500 \mathrm{~nm}, \mathrm{Cr}: 100 \mathrm{~nm}$ ), $\mathrm{C}_{60}$ 膜厚约 $370 \mathrm{~nm}$ (见图 1), 样品制备后在室温和低真空环境下避光保存.

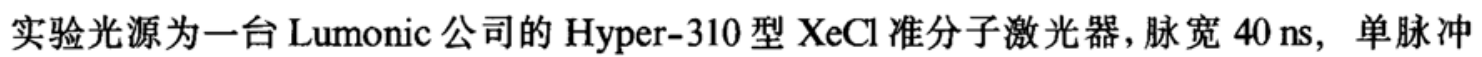
能量 $300 \mathrm{~mJ}$, 脉冲能量波动小于 $10 \%$. 为了使样品表面得到均匀的照射, 准分子激光器的输 出光束首先经过一个光束均匀器. 均匀器为口径 $5 \mathrm{~mm} \times 5 \mathrm{~mm}$, 长 $30 \mathrm{~cm}$ 的矩形波导, 内表面镀 铝以降低传输损耗. 对 “波导型均匀器”的详细描述见文献[5]. 输出的光强均匀面被成象于 样品表面, 能流密度通过插人衰减片或移动成象透镜改变均匀面大小进行调节. 用 38 单位的 一维紫外光电二极管阵列测得均匀面上的光强波动小于 $2 \%$.

首先用能量透射方法, 测量了空气中 $308 \mathrm{~nm}$ 准分子激光对 $\mathrm{C}_{600}$ 薄膜的刻蚀速率曲线和刻 蚀阈值. 有关实验的详细内容见文献[6]. 实验结果如图 2 所示, 刻蚀阈值约为 $36 \mathrm{~mJ} / \mathrm{cm}^{2}$.

然后用低于刻蚀阈值的激光脉冲照射薄膜样品, 用精度 $10^{-10} \mathrm{~A}$ 的检流计测量两极间的电 流, 电源电压 $1 \sim 12 \mathrm{~V}$.

原始 $C_{60}$ 薄膜和照射后样品的 Raman 谱用一台 Nicolet 公司的 950 型 Raman 谱仪测量,

1995-06-27 收稿, 1995-11-28 收修改稿 


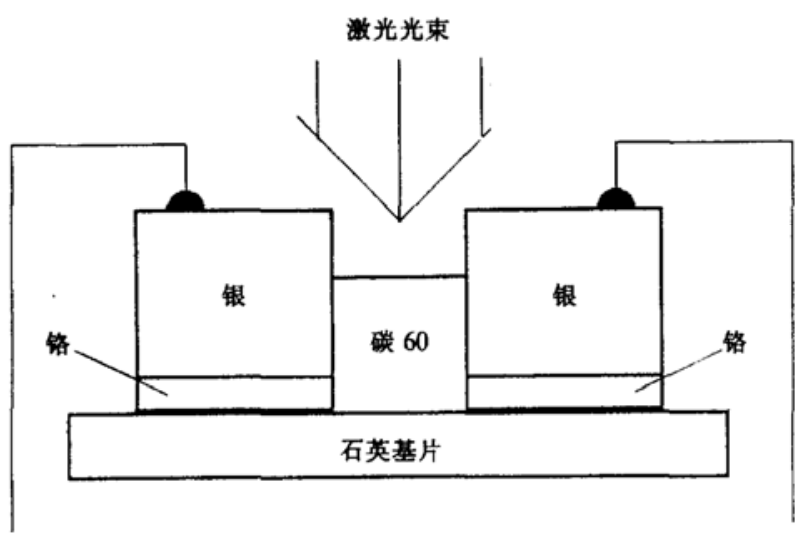

图 1 电导率实验中 $\mathrm{C}_{60}$ 薄膜样品的电极构型

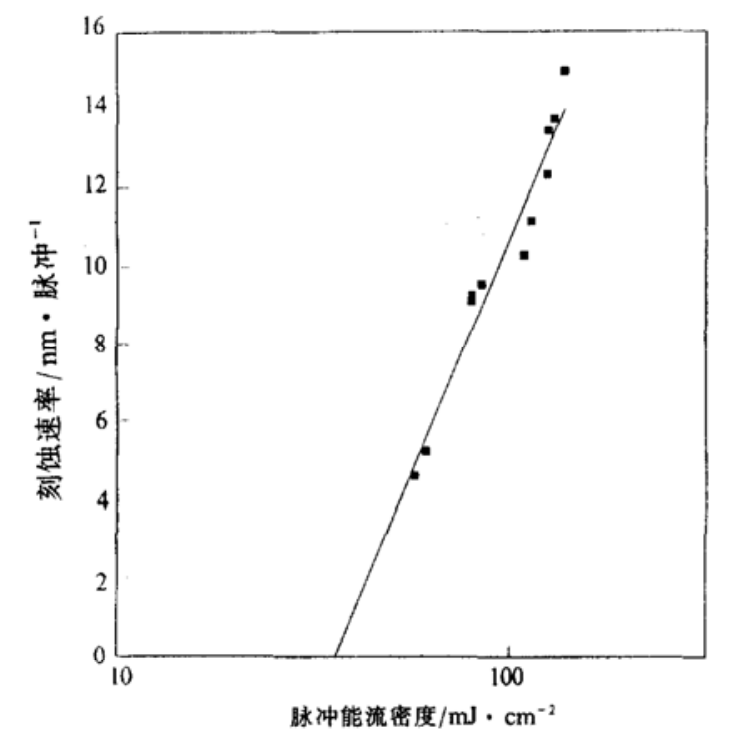

图 2 刻蚀速率随脉冲能流密度的变化 直线为数据的最小二次拟合, 假设满足 Beer 吸收定律, $\mathrm{C}_{60}$ 薄膜在 $308 \mathrm{~nm}$ 的吸收率约为 $9.6 \times 10^{5} \mathrm{~cm}^{-1}$. 刻蚀㜀值 $F_{\text {th }}=36 \mathrm{~mJ} / \mathrm{cm}^{2}$

激发波长 $1.06 \mu \mathrm{m}$.

\section{2 结果与讨论}

根据图 1 中的样品构形, 这里测量的实际为薄膜体电导率. 测得 $\mathrm{C}_{60}$ 薄膜的本征电导率约 为 $8.24 \times 10^{-8} \mathrm{~S} \cdot \mathrm{cm}^{-1}$, 与文献 [3] 中的 $6.4 \times 10^{-8} \mathrm{~S} \cdot \mathrm{cm}^{-1}$ 一致 (空气环境).

图 3 和图 4 分别给出在 $30 \mathrm{~mJ} / \mathrm{cm}^{2}$ 和 $34 \mathrm{~mJ} / \mathrm{cm}^{2}$ 能流密度的脉冲作用下, 样品的薄层电导 率 $\sigma_{\mathrm{d}}\left(\sigma_{\mathrm{d}}=\sigma \cdot d=\mathrm{S} \cdot l / w\right)$, 其中 $\sigma$ 为体电导率, $\mathrm{d}$ 为导电层厚度, $\mathrm{S}$ 为测量电导, $l$ 和 $w$ 为导电区长

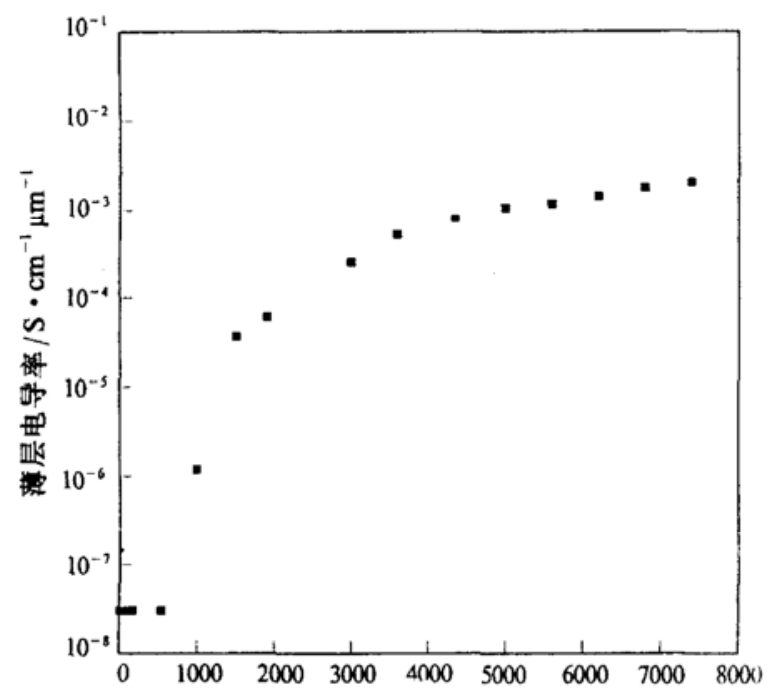

图 $330 \mathrm{~mJ} / \mathrm{cm}^{2}$ 时样品薄层电导率随照射脉冲数的 变化 (薄层电导率上升 5 个数量级)



图 $434 \mathrm{~mJ} / \mathrm{cm}^{2}$ 时样品薄层电导率随照射脉冲数的 变化 (薄层电导率上升 6 个数量级) 
宽) 随照射脉冲数的变化.

当能流密度为 $30 \mathrm{~mJ} / \mathrm{cm}^{2}$ 时, 样品电导率在约 540 个脉冲照射后开始上升约 5 个数量级, 5000 个脉冲后呈饱和趋势. 脉冲能流密度增大到 $34 \mathrm{~mJ} / \mathrm{cm}^{2}$ (略低于刻蚀阈值) 后, 电导率上 升速率急剧增大, 在 7 个脉冲后迅速上升 6 个数量级, 30 个脉冲后开始饱和. 由于脉冲能量 的波动 $(<10 \%)$, 此时可能有少量脉冲的能密度已超过了刻蚀阈值, 使薄膜发生少量刻蚀. 测量照射后薄膜样品的厚度, $30 \mathrm{~mJ} / \mathrm{cm}^{2}$ 时样品基本不变. 而 $34 \mathrm{~mJ} / \mathrm{cm}^{2}$ 时样品厚度减少约 $30 \mathrm{~nm}$.

原始 $\mathrm{C}_{60}$ 薄膜和激光照射后薄膜 样品的 Raman 的谱 $(1000 \sim 2000$ $\mathrm{cm}^{-1}$ ) 如图 5 所示. 原始 $\mathrm{C}_{60}$ 薄膜 Raman 谱呈现明显的 $\mathrm{C}_{60}$ 特征线 1469 $\mathrm{cm}^{-1}$ (Ag-symmetry "pentagonalpinch $\left.^{n} \operatorname{mode}^{(\eta)}\right)$, 及 $1500 \mathrm{~cm}^{-1}$ 处的宽 的对称峰, 后者为无定形石墨 $\left(\mathrm{sp}^{2}\right)$ 的 特征峰 ${ }^{[8]}$.

在 $30 \mathrm{~mJ} / \mathrm{cm}^{2}$ 的准分子激光脉冲 照射后, Raman 谱发生了较大的变 化:

(1) 出现了代表微晶石墨特征的 D 带 $\left(1320 \mathrm{~cm}^{-1}\right)$ 和 $\mathrm{G}$ 带 $(1580$ $\left.\mathrm{cm}^{-1}\right)^{[8 \sim 10]} \mathrm{G}$ 带为石墨的 $\mathrm{C}=\mathrm{C}$ 双键 拉伸振动模 $\left(\mathrm{E}_{2 \mathrm{~g}}\right)$, 而 $\mathrm{D}$ 带则源于小石 墨晶粒中缺乏长程有序导致的 $\kappa$ - 动 量守恒法则的破缺. D 带与 $G$ 带的

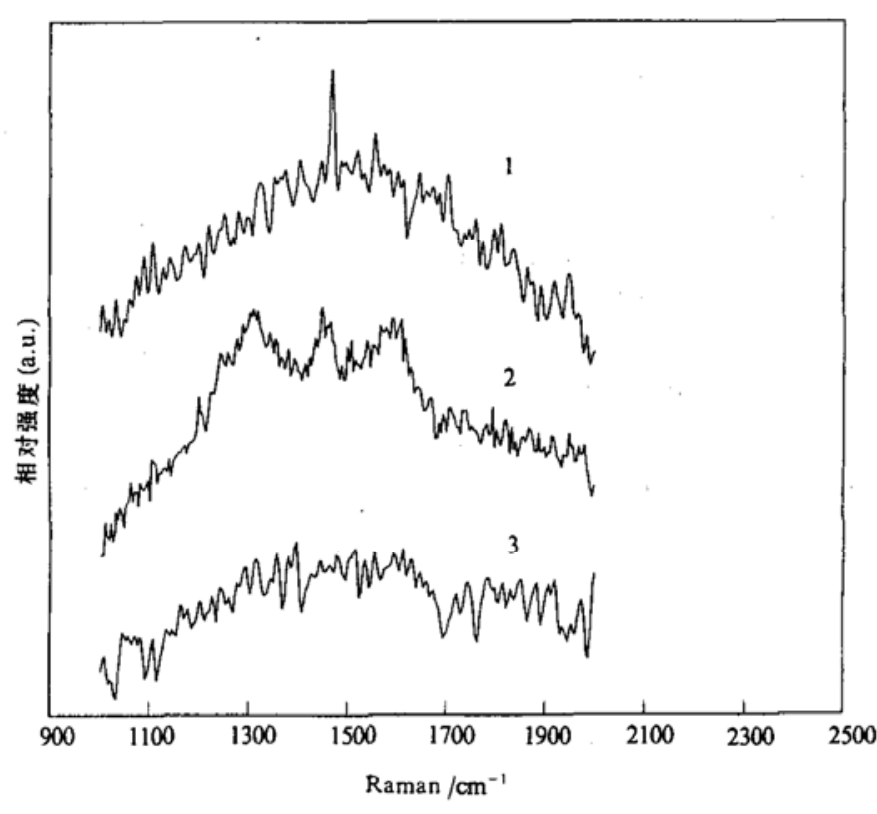

图 5 原始 $\mathrm{C}_{60}$ 薄膜和激光照射后样品的 Raman 谱 $1-\mathrm{C}_{60}$ 特征峰 $1469 \mathrm{~cm}^{-1}, 2-1500 \mathrm{~cm}^{-1}$ 处的宽的对称峰, 3 - 一无定型石墨 $\left(\mathrm{sp}^{2}\right)$ 的特征峰 积分强度的比值 $R=I(\mathrm{D}) / I(\mathrm{G})$ 与石墨晶粒尺度 $L_{\mathrm{a}}$ 成反比: $L_{\mathrm{a}} \approx 4.4[I(\mathrm{D}) / I(\mathrm{G})]^{-1[8,9}$. 由图 5 可推出 $30 \mathrm{~mJ} / \mathrm{cm}^{2}$ 时 $R=1.6$, 即晶粒尺度小于 $3 \mathrm{~nm}$.

(2) $\mathrm{C}_{60}$ 的特征峰被展宽, 峰值位置下移到 $1458 \mathrm{~cm}^{-1}$. 这一现象被 $\mathrm{Rao}$ 等证实为 $\mathrm{C}_{60}$ 的光致聚合 ${ }^{[11]}$. 在低强度紫外光作用下 $\mathrm{C}_{60}$ 发生聚合反应但氧的存在会阻碍这一过程的发 生. 在我们的实验中, 由于氧的存在, 使得光致聚合反应不可能在薄膜表面发生. 但由于石 墨表面层的形成, 阻碍了氧向薄膜内部的扩散. 因此, 薄膜内部的 $\mathrm{C}_{60}$ 分子在透过表面层的紫 外辐射作用下发生了光致聚合反应.

在 $34 \mathrm{~mJ} / \mathrm{cm}^{2}$ 时的 Raman 谱中, $C_{60}$ 特征峰消失, 只存在 $1500 \mathrm{~cm}^{-1}$ 处宽的对称峰. 因 此, 薄膜表面的 $\mathrm{C}_{60}$ 分子已被完全氧化分解为无定形碳, 而薄膜内部 $\mathrm{C}_{60}$ 分子的 Raman 信号则 被表面的无定形碳层完全吸收. 根据文献[6] 测得的 $\mathrm{C}_{60}$ 薄膜对 $308 \mathrm{~nm}$ 的吸收率 $9.6 \times 10^{5} \mathrm{~cm}^{-1}$, 吸收深度约为 $10 \mathrm{~nm}$. 假设这一深度即为导电层厚度, 则 $34 \mathrm{~mJ} / \mathrm{cm}^{2}$ 照射后, 样品体电导率约 为 $1 \mathrm{~S} \cdot \mathrm{cm}^{-1}$, 与无定形碳的电导率基本一致 ${ }^{\left[{ }^{[1]}\right.}$. 


\section{参考文献}

I Schumann M, Sauerbrey R, Smayling M C. Permanent increase of the electrical conductivity of polymers induced by ultraviolet laser irradiation. Appl Phys Lett, 1991, 58(5): $428 \sim 431$

2 Satio S, Oshiyarna A. Cohesive mechanism and energy bands of solid $\mathrm{C}_{60}$. Phys Rev Lett, 1991, 66(20): $2637 \sim 2640$

3 Hamed A, Sun $\mathrm{Y}$, Tao $\mathrm{Y}$ et al. Effects of oxygen and illumination on the in situ conductivity of $\mathrm{C}_{60}$ thin films. Phys Rev, 1993, B47(16): $10873 \sim 10880$

4 Phillips H M, Serkar D, Halas N J et al. Excimer-laser-induœed electrical conductivity in thin-film $\mathrm{C}_{60}$. Appl Phys, 1993, A57(1): 105 107

5 Brannon J H. Micropatterning of the surfaces by excimer laser projection. J Vac Sci Technol, 1989, B7(5): $1064 \sim 1071$

6 宁 东, 楼棋洪, 叶震蓑等. $308 \mathrm{~nm}$ 准分子激光对 $\mathrm{C}_{60}$ 薄膜的刻蚀特征研究. 光学学报, 1995, 15(7): $909 \sim 912$

7 Zhou Ping, Wang Kaian, Wang Ying et al. Raman scattering in $\mathrm{C}_{60}$ and alkali-metal-saturated $\mathrm{C}_{60}$. Phys Rev, 1992, B46(4): $2595 \sim 2605$

8 Nistor L C, Vanlanduyt J, Ralchenko V G et al. Direction observation of laser-induced-crystallization of a-C: $\mathrm{H}$ films. Appl Phys, 1994, A58(1): 137 144

9 Dillon R O, Woolam J A. Use of Raman scattering to investigate disorder and crystallite formation in as-deposited and annealed carbon films. Phys Rev, 1984, 29(6): $3482 \sim 3489$

10 Nemanich R J, Class J T, Lucovsky G et al. Raman scattering characterization of carbon bonding in diamond and diamondlike thin films. J Vac Sci Technol, 1988, A6(3): $1783 \sim 1787$

11 Rao A M, Zhou Ping, Wang Kaian et al. Photo-induced polymerization of solid $\mathrm{C}_{60}$ films. Science, 1993, 259: 955 $\sim 957$ 